\title{
Percepción de la imagen corporal en una muestra de universitarios de la ciudad de Medellín ${ }^{*}$
}

\section{Perception of body image in university students of the Medellín City}

\author{
e \\ Dubis Marcela Rincón Barreto, Carolina Suárez Martínez, \\ Sara Mesa Benítez, Angie Carolina Orozco Martínez, \\ Juan Diego López Botero, Juan David Marín Hoyos, Ruth \\ Daniris Murillo Mosquera, Sara Camila Narvaez Roldan, \\ Juan Pablo Castaño Lezcano, María Camila Navarrete
}

* Artículo producto de una investigación, Semillero
Adicciones y Salud Mental, Universidad Católica Luis
Amigó
Magister en Neuropsicología Clínica. Docente Uni-
versidad Católica Luis Amigó. Medellín - Colombia,
ORCID: 0000-0002-8322-889X, dubis.rinconba@amigo.
edu.co
Estudiantes de Psicología de la Universidad Católica
Luis Amigó. Semillero Adicciones y Salud Mental, ca-
rolina.suarezma@amigo.edu.co
Estudiante de Psicología de la Universidad Católica
Luis Amigó. Semillero Adicciones y Salud Mental,
sara.mesaen@amigo.edu.co
Estudiante de Psicología de la Universidad Católica
Luis Amigó. Semillero Adicciones y Salud Mental, an-
gie.orozcoma@amigo.edu.co

Estudiante de Psicología de la Universidad Católica Luis Amigó. Semillero Adicciones y Salud Mental, juan.lopezot@amigo.edu.co

Estudiante de Psicología de la Universidad Católica Luis Amigó. Semillero Adicciones y Salud Mental, juan.marinho@amigo.edu.co

Estudiante de Psicología de la Universidad Católica Luis Amigó. Semillero Adicciones y Salud Mental, ruth.murillomo@amigo.edu.co

Estudiante de Psicología de la Universidad Católica Luis Amigó. Semillero Adicciones y Salud Mental, sara.narvaezro@amigo.edu.co

Estudiante de Psicología de la Universidad Católica Luis Amigó. Semillero Adicciones y Salud Mental, juan.castanole@amigo.edu.co

Estudiante de Psicología de la Universidad Católica Luis Amigó. Semillero Adicciones y Salud Mental, maria.navarrete@amigo.edu.co
Recibido 14.11. 2019• Arbitrado 02.12. $2019 \bullet$

Aprobado 13.12. 2019

\section{Resumen}

El objetivo de este estudio es describir la percepción sobre la imagen corporal de universitarios de la ciudad de Medellín. Para ello, se realizó una investigación de tipo descriptivo con un diseño transversal y se contó con una muestra de 452 estudiantes (123 hombres y 329 mujeres) de las diferentes facultadas de la Universidad Católica Luis Amigó a quienes se les aplicó el Cuestionario Multidimensional sobre la Imagen Corporal (MBSRQ) (Ribas, Botella y Benito, 2008) el cual evalúa 4 componentes de la imagen corporal: (1) importancia subjetiva de la corporalidad (ISC); conductas 
orientadas a mantener la forma física (COMF); (3) atractivo físico autoevaluado (AFA); y (4) cuidado del aspecto físico (CAF). Como principales resultados se encontró que las personas suelen considerar importante la realización de conductas para mantenerse en forma, pero esto no se relaciona con la valoración sobre el atractivo físico percibido. Así mismo, se evidenció que las personas que valoran positivamente el cuidado del aspecto físico, también le otorgan importancia a la corporalidad y al desarrollo de conductas que les permitan mantener adecuadamente su aspecto físico.

Palabras clave: imagen corporal, percepción, estudiante universitario, apariencia física.

\section{Abstract}

The objective of this study is to describe the perception of the body image of university students in the city of Medellín. For this, a descriptive type investigation was carried out with a cross-sectional design and a sample of 452 students (123 men and 329 women) from the different faculties of the Luis Amigó Catholic University were given to whom the Multidimensional Questionnaire on the Body Image (MBSRQ) (Ribas, Botella and Benito, 2008) which evaluates 4 components of body image: (1) subjective importance of corporality (SIC); behaviors aimed at maintaining physical fitness (BMF); (3) self-assessed physical attractiveness (SFA); and (4) physical appearance care (FAC). As main results it was found that people often consider it important to carry out behaviors to keep fit, but this is not related to the assessment of perceived physical attractiveness. Likewise, it was evidenced that people who value positively the care of the physical aspect, also gives importance to the corporality and the development of behaviors that allow them to properly maintain their physical appearance.

Keywords: body image, perception, university student, physical appearance.

\section{Introducción}

La imagen corporal, es un constructo que implica lo que uno piensa, siente y cómo actúa en relación al propio cuerpo (Baile, Raich \& Garrido, 2003; Cash, 2004). En términos generales, es una representación consciente y un conocimiento conceptual acerca del cuerpo (Meneses \& Moncada, 2008; Botella, Grañó, Gámiz, \& Abey, 2008; Restrepo \& Castañeda, 2018) la cual, conforme aparecen nuevas formas de comunicación e interrelación, se inserta como una valoración y una construcción intersubjetiva. La percepción de la imagen corporal es una construcción social, permeada por la cultura (costumbres, hábitos) de cada sociedad. Por ejemplo, en Asia, a las mujeres desde la infancia se les provocan atrofias en los pies, para hacerlos más pequeños y deformes; 
así mismo, en algunas tribus de África, exhiben largos cuellos como de jirafa, en consecuencia, del uso permanente de accesorios o aros alrededor de este (De Pedro, 2004)

De otro lado, Raich (2004) señala que la imagen corporal es una representación mental del propio cuerpo y desde un punto de vista neurológico, constituye una representación mental diagramática de la conciencia corporal de cada persona. Por tanto, las alteraciones de la imagen corporal serían el resultado de una perturbación en el funcionamiento cortical.

Actualmente, la susceptibilidad de la imagen corporal ocupa un rol significativo debido a los medios masivos de comunicación, representados esencialmente por la Internet y las redes sociales. Tal como afirma Cortez et al. (2016), la vertiginosa y vasta difusión de patrones y conductas valoradas como positivas, por ser consideradas equivalentes de éxito, belleza, atractivo, inteligencia y salud, impactan en mayor medida a poblaciones vulnerables que ceden a las presiones socioculturales, encontrando que el uso de Internet provoca un aumento en la insatisfacción corporal en ambos sexos (Angelakis, Gooding \& Panagioti, 2016). En palabras de Ramos-Vidal (2019) las personas tienden a ajustar su comportamiento al del grupo de iguales para buscar la aprobación y evitar la exclusión del grupo.

Articulado a esto, hay otros factores predisponentes tales como el modelo familiar, aspectos personales que comprenden desde la percepción de sí mismo hasta la presencia de un trastorno asociado con la distorsión de la imagen corporal, el desarrollo físico a partir de la evaluación individual del aspecto en cada etapa evolutiva y los posibles eventos traumáticos que pudieron originar un cambio radical en la autopercepción corporal (Salaberria, Rodríguez \& Cruz, 2007).

De esta manera, es posible evidenciar que, ante las nuevas configuraciones del cuerpo y su imagen, emergen también otras formas de psicopatología, las cuales, aunque han estado presente desde hace aproximadamente dos siglos (Berrios, 1996), actualmente configuran altas tasas de prevalencia, impactando significativamente la salud mental (OMS, 2013). Por ejemplo, es el caso del dismórfico corporal (TDC), el cual comprende una distorsión de la percepción de la imagen corporal y según el DSM 5, se define como la preocupación excesiva por los defectos o imperfecciones físicas (APA, 2014). Por lo que la imagen corporal o representación mental del propio cuerpo adquiere gran relevancia para el desarrollo de dicho trastorno (Giraldo-O'Meara \& Belloch, 2017).

La presente investigación tiene como objetivo analizar la percepción de la imagen corporal y su relación con algunas variables sociodemográficas en una 
muestra de universitarios de la ciudad de Medellín a partir del Cuestionario Multidimensional sobre la imagen corporal (MBSRQ) (Ribas, Botella \& Benito, 2008).

\section{Metodología}

La investigación se desarrolló bajo un enfoque cuantitativo, con diseño metodológico de tipo descriptivo y de corte transversal. Según Hernández, Fernández-Collado y Baptista (2006) una investigación de tipo descriptiva pretende especificar las propiedades, las características y los perfiles de personas, grupos, comunidades, procesos, objetos o cualquier otro fenómeno que se someta a un análisis.

\section{Participantes}

Se contó con una muestra de 452 universitarios de la ciudad de Medellín, con edades comprendidas entre los 16-55 años de edad (27,2 \% hombres y $72,8 \%$ mujeres), adscritos a las diferentes facultades de la Universidad Católica Luis Amigó. En cuanto a la talla y el peso, la media para las mujeres fue de 160 centímetros (cms) y 64.9 kilogramos (Kgs) respectivamente; en el caso de los hombres fue de $163 \mathrm{cms}$ y $64.9 \mathrm{kgs}$.

Se tuvo en cuenta como criterios de inclusión, que fueran miembros activos de la comunidad universitaria y que aceptaran mediante consentimiento informado (virtual) la participación en el estudio y el tratamiento de sus datos. En la tabla 1 se muestran los datos sociodemográficos y clínicos de los participantes.

Tabla 1. Datos Sociodemográficos y clínicos

\begin{tabular}{cccc}
\hline & Descripción & Total & Porcentaje \\
\hline \multirow{2}{*}{ Sexo } & Hombre & 123 & 27,2 \\
& Mujer & 329 & 72,8 \\
\hline \multirow{6}{*}{ Facultad } & CACE & 52 & 11,5 \\
& COMPUB & 71 & 15,7 \\
& DERECHO & 39 & 8,6 \\
& EDUYHUM & 76 & 16,8 \\
& INGEYARQUI & 14 & 3,1 \\
& PSICOLOGIA & 200 & 44,2 \\
\hline
\end{tabular}




\begin{tabular}{cccc}
\hline & Descripción & Total & Porcentaje \\
\hline \multirow{2}{*}{ Asiste al Gimnasio } & Si & 56 & 12,4 \\
& No & 369 & 81,6 \\
\hline & Alcohol & 12 & 2,7 \\
Consumo de SPA & Cannabis & 13 & 2,9 \\
& No consume & 423 & 93,6 \\
& Otro & 4 & 0,9 \\
\hline \multirow{2}{*}{ Talla } & Hombre & $163 \mathrm{cms}$ & \\
& Mujer & $160 \mathrm{cms}$ & \\
\hline \multirow{2}{*}{ Peso } & Hombre & $64,9 \mathrm{kgs}$ & \\
& Mujer & $64,9 \mathrm{kgs}$ & \\
\hline
\end{tabular}

Fuente: Los autores

\section{Instrumentos}

Cuestionario Multidimensional sobre la Imagen Corporal (MBSRQ) (Ribas, Botella y Benito, 2008), instrumento que evalúa aspectos cognitivos y conductuales, valora la insatisfacción corporal y discrimina las ideas sobrevaloradas acerca de la apariencia. Se utilizó la versión española que consta de 45 ítems, los cuales pueden agruparse en 4 factores que describen: 1) la Importancia Subjetiva de la Corporalidad (ISC), 2) Conductas Orientadas a Mantener la Forma Física (COMF), 3) Atractivo Físico Autoevaluado (AFA), 4) Cuidado del Aspecto Físico (CAF). En cuanto a la fiabilidad del cuestionario, su consistencia interna es igual a un índice de Alfa de Cronbach de 0,884 $(a=0,884)$.

\section{Procedimiento}

Los datos se recolectaron mediante la aplicación del Cuestionario Multidimensional sobre la imagen corporal (MBSRQ) Botella, Ribas \& Benito-Ruiz (2009) cual fue digitalizado y compartido mediante un formulario de google, en el cual se incluyó el consentimiento informado y la aceptación de participación. Para el envío del formulario se utilizó la cuenta oficial de la Universidad para el envío de correos masivos, con el fin de garantizar la protección de los datos.

Para el análisis de los datos, se empleó el paquete estadístico SPSS versión 25 de 2018. Los datos sociodemográficos se analizaron con medidas de estadística descriptiva (media) y para el análisis de las respuestas al cuestionario, 
se emplearon medidas no paramétricas (Coeficiente de Spearman), al determinarse que no se trataba de una muestra con distribución normal.

\section{Resultados}

Como se presenta en la tabla 2 , el factor 2 cuyas preguntas están relacionadas con: conductas orientadas a mantener la forma física, se encuentra relacionado con el sexo, el peso y la asistencia al gimnasio, aunque no se precisan diferencias entre los sexos. En cuanto al factor 3: Atractivo Físico Autoevaluado (AFA), relacionado con la autoevaluación del atractivo físico, se evidenció una correlación positiva con la edad y la asistencia al gimnasio. Respecto al factor cuatro: 4) Cuidado del Aspecto Físico (CAF), el cual evaluó el cuidado del aspecto físico solo se encontró relacionado con la variable peso, mientras que el factor 1: importancia Subjetiva de la Corporalidad, no presentó relación con alguna de las variables sociodemográficas de la muestra.

Tabla 2. Relación de los factores con los datos sociodemográficos

\begin{tabular}{|c|c|c|c|c|c|c|c|c|}
\hline & Datos & Sociodemográficos & Edad & Facultad & Sexo & $\begin{array}{c}\text { Talla } \\
\text { (cms) }\end{array}$ & $\begin{array}{l}\text { Peso } \\
(\mathrm{kg})\end{array}$ & $\begin{array}{l}\text { Asiste al } \\
\text { Gimnasio }\end{array}$ \\
\hline \multirow{9}{*}{ 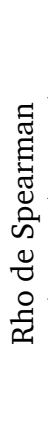 } & Factor & Coeficiente de correlación & 0,001 & 0,02 & 0,029 & $-0,019$ & $-0,089$ & $-0,064$ \\
\hline & 1 & Sig. (bilateral) & 0,984 & 0,664 & 0,533 & 0,703 & 0,058 & 0,178 \\
\hline & Factor & Coeficiente de correlación & $-0,015$ & $-0,006$ &, $133^{* *}$ & 0,025 &,$- 098^{*}$ &,$- 214^{* *}$ \\
\hline & 2 & Sig. (bilateral) & 0,751 & 0,904 & 0,005 & 0,61 & 0,038 & 0 \\
\hline & Factor & Coeficiente de correlación & ,105* & $-0,002$ & $-0,011$ & $-0,051$ & $-0,092$ & , $144^{* * *}$ \\
\hline & 3 & Sig. (bilateral) & 0,025 & 0,968 & 0,812 & 0,297 & 0,051 & 0,002 \\
\hline & \multirow{3}{*}{$\begin{array}{c}\text { Factor } \\
4\end{array}$} & Coeficiente de correlación & 0,019 & $-0,066$ & $-0,017$ & $-0,006$ &,$- 137^{* *}$ & $-0,072$ \\
\hline & & Sig. (bilateral) & 0,68 & 0,161 & 0,712 & 0,909 & 0,003 & 0,126 \\
\hline & & $\mathrm{N}$ & 452 & 452 & 452 & 413 & 452 & 452 \\
\hline
\end{tabular}

*. La correlación es significativa en el nivel 0,05 (bilateral).

**. La correlación es significativa en el nivel 0,01 (bilateral).

Fuente: IBM SPSS Statistics editor de datos, versión 25, 2018

En cuanto a la asociación entre los factores, como se expresa en la tabla 3, se encontró que el factor 1 se encuentra relacionado con el factor 2 y 4, es decir, que la importancia subjetiva de la corporalidad, se relacionan positivamente con las conductas orientadas a mantener la forma física y el cuidado del aspecto físico. Lo que sugiere que el desarrollo de actividades relacionadas con el cuidado del cuerpo da cuenta de la importancia que este representa para las personas. De esta manera el factor 2, se relaciona positivamente con 
el 1 y el 4, mientras que no con el factor 3, relacionado con el atractivo físico autoevaluado, lo que llama la atención, en tanto sugiere que una persona puede desarrollar conductas para mantenerse en forma, aunque no valore positivamente el atractivo físico.

Respecto al factor 4: cuidado del aspecto físico, correlaciona positivamente con el factor 1, 2 y 3 . En este orden, una persona que valorar positivamente el cuidado del aspecto físico, también le otorga importancia a la corporalidad y al desarrollo de conductas que le permitan mantener adecuadamente su aspecto físico.

Tabla 3. Relación entre factores

\begin{tabular}{|c|c|c|c|c|c|c|}
\hline & & & Factor 1 & Factor 2 & Factor 3 & Factor 4 \\
\hline \multirow{12}{*}{ 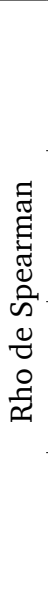 } & \multirow{3}{*}{ Factor 1} & Coeficiente de correlación & 1 & ,433 $3^{* *}$ & 0,066 & ,306 \\
\hline & & Sig. (bilateral) & . & 0 & 0,159 & 0 \\
\hline & & $\mathrm{N}$ & 452 & 452 & 452 & 452 \\
\hline & \multirow{3}{*}{ Factor 2} & Coeficiente de correlación &, $433^{* *}$ & 1 & 0,007 & ,322 \\
\hline & & Sig. (bilateral) & 0 & . & 0,876 & 0 \\
\hline & & $\mathrm{N}$ & 452 & 452 & 452 & 452 \\
\hline & \multirow{3}{*}{ Factor 3} & Coeficiente de correlación & 0,066 & 0,007 & 1 &, $133^{* *}$ \\
\hline & & Sig. (bilateral) & 0,159 & 0,876 & . & 0,005 \\
\hline & & $\mathrm{N}$ & 452 & 452 & 452 & 452 \\
\hline & \multirow{3}{*}{ Factor 4} & Coeficiente de correlación &, $306^{* *}$ &, $322^{* *}$ &, $133^{* *}$ & 1 \\
\hline & & Sig. (bilateral) & 0 & 0 & 0,005 & . \\
\hline & & $\mathrm{N}$ & 452 & 452 & 452 & 452 \\
\hline
\end{tabular}

**. La correlación es significativa en el nivel 0,01 (bilateral).

Fuente: IBM SPSS Statistics editor de datos, versión 25, 2018

\section{Discusión y conclusiones}

La imagen corporal, vista como una valoración que cada individuo realiza sobre el cuerpo, involucra, como todo proceso actitudinal, aspectos cognitivos, emocionales y comportamentales, entre los que se puede destacar la conducta auto reflexiva frente al lugar preponderante que puede adquirir una parte del cuerpo o el significado de este como parte del proceso de socialización (Sánchez \& López, 2013). La imagen corporal se transforma conforme aparecen nuevas formas de comunicación e interrelación, por ejemplo, el uso de los dispositivos digitales y las redes sociales, generando una sobrevaloración de la opinión del 
otro sobre el propio cuerpo y una creciente necesidad de aprobación (Pistoia, Carolei, Sacco \& Sarà, 2017).

Tal como lo afirman, Moreno y Ortiz (2009) la preocupación por el peso y la insatisfacción por la imagen corporal constituyen los dos elementos básicos de una baja autoestima, con base en inferencias y creencias, muchas de ellas sujetas a distorsiones perceptuales.

Según Ramos-Vidal (2019), algunos cambios en el comportamiento, como por ejemplo, ir a un gimnasio y generar cambios en los hábitos de alimentación se relacionan la búsqueda de aprobación de un grupo. No obstante, los resultados de la investigación, demostraron disociaciones entre los componentes cognitivos (lo que piensan), conductuales (lo que hacen) y emocionales (lo que sienten) de la imagen corporal. De esta manera, las personas evaluadas valoraron positivamente la percepción u opinión de los demás pero esto no se asoció significativamente con la importancia subjetiva de la corporalidad. Así mismo, los participantes valoraron positivamente aspectos como el atractivo físico, el cuidado del peso y las conductas para mantener una buena apariencia, aunque esto no se relacionó con la realización de actividad o ejercicio físico. Es decir, no por considerar importante el atractivo, esto conlleva a una conducta de cuidado o mantenimiento físico. Por ejemplo, las personas que reportaron insatisfacción con su cuerpo, indicaron no asistir al gimnasio.

Finalmente, tal como lo menciona Rincón-Barreto (2019) para hablar del cuerpo, es necesario recurrir a la experiencia individual y colectiva que se tiene de este, a su construcción social y al nivel de satisfacción o aprobación que sea capaz de proporcionar. Así las cosas, el cuerpo y específicamente la imagen corporal, adquiere una relevancia especial en tanto da cuenta de la cultura y las prácticas sociales contemporáneas. La imagen corporal, es un constructo que implica lo que uno piensa, siente y cómo actúa en relación a su propio cuerpo. En términos generales, es una representación consciente y un conocimiento conceptual acerca del cuerpo (Meneses et al., 2008).

\section{Agradecimientos}

A la Universidad Católica Luis Amigó y su Oficina de Comunicaciones, a través de la cual se convocó la población a partir de la cual se realizó la selección de la Muestra. 


\section{Referencias}

American Psychiatric Association (APA) (2014). DSM-5. Manual Diagnóstico y Estadístico de los Trastornos Mentales. Buenos Aires: Editorial Médica Panamericana.

Angelakis, I., Gooding, P., Panagioti, M. (2016). Suicidality in body dysmorphic disorder (BDD): A systematic review with meta-analysis. Clin Psychol Rev., 49:55-66. Doi: 10.1016/j.cpr.2016.08.002.

Baile, J. I., Raich, R., Garrido, E. (2003). Evaluación de insatisfacción corporal en adolescentes: efecto de la forma de administración de una escala. Anales de Psicología/Annals of Psychology, 19(2), 187-192, https://www.um.es/analesps/ v19/v19_2/02-19_2.pdf

Berrios, G. (1996). La historia de los síntomas de los trastornos mentales, la psicopatología descriptiva desde el siglo XIX. Universidad de Cambridge. ISSN 9786071615633.

Botella, L., Grañó, N., Gámiz, M. y Abey, M. (2008). La Presencia Ignorada del Cuerpo: Corporalidad y (re)construcción de la identidad. Revista Argentina de Clínica Psicológica, XVII (3), 245-263, https://www.redalyc.org/ pdf/2819/281921795006.pdf

Botella, L. Ribas, E., Benito-Ruiz, J. (2009). Evaluación psicométrica de la imagen corporal: validación de la versión española del Multidimensional Body Self Relations Questionnaire (MBSRQ). Revista Argentina de Clinica Psicologica. XVIII (3), 253-264, https://www.redalyc.org/articulo.oa?id=281921775006

Cash, T. F. (2004). Body image: past, present, and future. Body Image, 1(1), 1-5. https://doi.org/10.1016/s1740-1445(03)00011-1

Cortez, D., Gallegos, M., Jiménez, T., Martínez, P., Saravia, S., Cruzat-Mandich, C., Arancibia, M. (2016). Influencia de factores socioculturales en la imagen corporal desde la perspectiva de mujeres adolescentes. Revista Mexicana de Trastornos Alimentarios, 7(2), 116-124. Doi: https://dx.doi.org/10.1016/j.rmta.2016.05.001

De Pedro, I. D. L. S. (2004). Introducción: alteraciones de la imagen corporal. Monografías de psiquiatría, 16(2), 1-2. https://dialnet.unirioja.es/servlet/ articulo? codigo $=1148300$

Giraldo-O’Meara, M., Belloch, A. (2017). El trastorno dismórfico corporal: Un problema infradiagnosticado. Revista de Psicopatología y Psicología Clínica, 22, 6984, Doi: 10.5944/rppc.vol.22.num.1.2017.17929

Hernández Sampieri, R. Fernández Collado, C., Baptista Lucio, P. (2014). Metodología de la investigación (5a. ed.). México D.F. 
Meneses, M., Moncada, J. (2008). Imagen corporal percibida e imagen corporal deseada en estudiantes universitarios costarricenses. Revista Iberoamericana de Psicología del Ejercicio y el Deporte, 3(1), 13-30, Disponible en: http://www. redalyc.org/articulo.oa?id $=311126259002$

Organización Mundial de la Salud-OMS (2013). Plan de acción sobre salud mental 2013-2020. Disponible en https://www.who.int/mental_health/publications/ action_plan/es/

Pistoia, F., Carolei A., Sacco, S., Sarà, M. (2017) Commentary: Embodied Medicine: Mens Sana in Corpore Virtuale Sano. Front. Hum. Neurosci. 11:381. Doi: 10.3389/ fnhum.2017.00381

Ramos-Vidal I (2019) El papel de las relaciones y la satisfacción vital en la percepción de la auto-imagen en una muestra de adolescentes mexicanos. Arch Med (Manizales); 19(1), 111-23. DOI: https://doi.org/10.30554/arch-med.19.1.2742.2019.

Raich, R. (2004). Una perspectiva desde la psicología de la salud de la imagen corporal. Avances en Psicología Latinoamericana, 22(1), 15-27. Recuperado de https://revistas.urosario.edu.co/index.php/apl/article/view/1261

Restrepo, E., Castañeda, L. (2018). Riesgo de trastorno de la conducta alimentaria y uso de redes sociales en usuarias de gimnasios de la ciudad de Medellín, Colombia. Revista Colombiana de Psiquiatría, 47(4). Doi: 10.1016/j.rcp.2018.08.003

Rincón-Barreto, D. (2019). En la era hegemónica del cuerpo, una pausa para comprender el trastorno dismórfico corporal. Drugs and Addictive Behavior, 4(2), 1-4, DOI: https://doi.org/10.21501/24631779.3366

Salaberria, K., Rodríguez, S., Cruz, S. (2007). Percepción de la imagen corporal. Osasunaz, 8(2), 171-183, http://www.euskomedia.org/PDFAnlt/ osasunaz/08/08171183.pdf

Sánchez-Núñez, R., López-Silva, U. (2013). Belleza y psicopatología. Reporte de un caso. Revista Medicina Universitaria, 15 (60), 129-134, https://www.elsevier.es/ en-revista-medicina-universitaria-304-pdf-X1665579613496148 